\title{
Importance of pulmonary valve morphology for pulmonary valve preservation in tetralogy of Fallot surgery: compari- son of the echocardiographic parameters
}

Su Jin Choi, $M D^{1}$, Jung Eun Kwon, MD¹, Da Eun Roh, MD¹, Myung Chul Hyun, MD, PhD ${ }^{1}$, Hanna Jung, MD, PhD², Young Ok Lee, MD, PhD², Joon Yong Cho, MD², Yeo Hyang Kim, MD, PhD ${ }^{1}$

'Department of Pediatrics, School of Medicine, Kyungpook National University, Division of Pediatric Cardiology, Kyungpook National University Children's Hospital, Daegu, Korea; ${ }^{2}$ Department of Thoracic and Cardiovascular Surgery, School of Medicine, Kyungpook National University, Daegu, Korea

Background: The decision to use transannular patching (TAP) during tetralogy of Fallot (TOF) repair depends on the pulmonary valve annulus size; the $z$ score of the pulmonary annulus is the most commonly used predictor. However, definitive results are not obtained with $z$ scores as different $z$ score data sets are used for different parameters.

Purpose: This study aimed to identify the echocardiographic and other key factors that warranted a change in the surgical method during TOF surgery.

Methods: Sixty-two patients were enrolled and divided into a pulmonary valve (PV) preservation group and a TAP group. Their medical records were reviewed.

Results: The $z$ score for PV annulus (PVA), ratio of the PVA to aortic annulus size, and ratio of PVA to descending aorta (DAO) size were significantly different between the $P V$ preservation and TAP groups $(-1.72 \pm 1.52$ vs. $-3.07 \pm 1.94, P=0.004 ; 0.62 \pm 0.12$ vs. $0.50 \pm 0.14, P=0.002$; and $1.32 \pm 0.32$ vs. $1.07 \pm 0.36, P=$ 0.008 , respectively). For TAP repair, the PVA $z$ score had a sensitivity of $65.4 \%$ and specificity of $73.1 \%$, ratio of PVA to aortic annulus size had a sensitivity of $73.1 \%$ and specificity of $65.4 \%$, and ratio of PVA to DAO size had a sensitivity of $69.2 \%$ and specificity of $57.7 \%$. The TAP group showed more monocuspid PVs $(P=0.011)$, while the PV preservation group showed more tricuspid PVs $(P=0.027)$. Commissurotomy was more frequently performed in the $\mathrm{PV}$ preservation group than in the TAP group ( $P=0.001)$. Of patients with commissurotomy, $58 \%$ showed a PV $z$ score $<-2$.

Conclusion: Although various echocardiographic parameters may serve as predictors for determining surgical methods for TOF patients, the PV morphology and tissue characteristics should also be considered.

Key words: Echocardiography, Pulmonary valve, Surgery, Tetralogy of Fallot

\section{Key message}

Question: Is echocardiographic examination is useful for surgical method decision in patients with tetralogy of Fallot (TOF)?

Finding: Various echocardiographic parameters serve as predictors for determining surgical methods for TOF patients. However, the pulmonary valve morphology and tissue characteristics should also be considered.

Meaning: A full dependence on the size of the pulmonary valve annulus (PVA), the $z$ score, and the ratio of the size of PVA to aortic valve annulus or descending aorta surgery may result in inadequate surgical results during TOF total correction.

\section{Introduction}

Closing the interventricular septal defect and relieving right ventricular outflow tract (RVOT) stenosis are key to surgical treatment of tetralogy of Fallot (TOF). ${ }^{1)}$ Although it is possible to mitigate RVOT stenosis while preserving the pulmonary valve $(\mathrm{PV})$ in some patients, a PV annulus (PVA) incision followed by transannular patch (TAP) repair is necessary in other patients. ${ }^{1,2)}$

Although TAP repair can efficiently alleviate RVOT stenosis, it is associated with increased postoperative mortality and morbidity in patients with TOF, as it may prolong pulmonary regurgitation, cause right ventricular (RV) enlargement, and induce RV failure. ${ }^{3,4)}$ In contrast, preserving the PV during TOF repair, wherein the PVA is preserved to maintain PV function, is beneficial with respect to reducing PV regurgitation and maintaining long-term RV function. ${ }^{5,6}$ Therefore, evaluating the need for TAP repair is crucial for patient prognosis.

The need for TAP repair is generally determined based on the $z$ score of the size of the PVA, which the cutoff value for this measure varies from -4 to -1 among studies. ${ }^{5,79}$ However, determining the need for TAP repair solely based on the $z$ score may be inadequate. Although it is a standardized index, the $z$ score is affected by various factors, such as height, weight, and body

\footnotetext{
Corresponding author: Yeo Hyang Kim, MD, PhD. Department of Pediatrics, School of Medicine, Kyungpook National University, 680 Gukchaebosang-ro, Jung-gu, Daegu 41944, Korea

凶E-mail: kimyhmd@knu.ac.kr, https://orcid.org/0000-0002-1631-574X

Received: 7 September, 2019, Revised: 6 November, 2019, Accepted: 6 November, 2019

This is an open-access article distributed under the terms of the Creative Commons Attribution Non-Commercial License (http://creativecommons.org/licenses/by$\mathrm{nc} / 4.0 /$ ) which permits unrestricted non-commercial use, distribution, and reproduction in any medium, provided the original work is properly cited.

Copyright (c) 2020 by The Korean Pediatric Society
} 


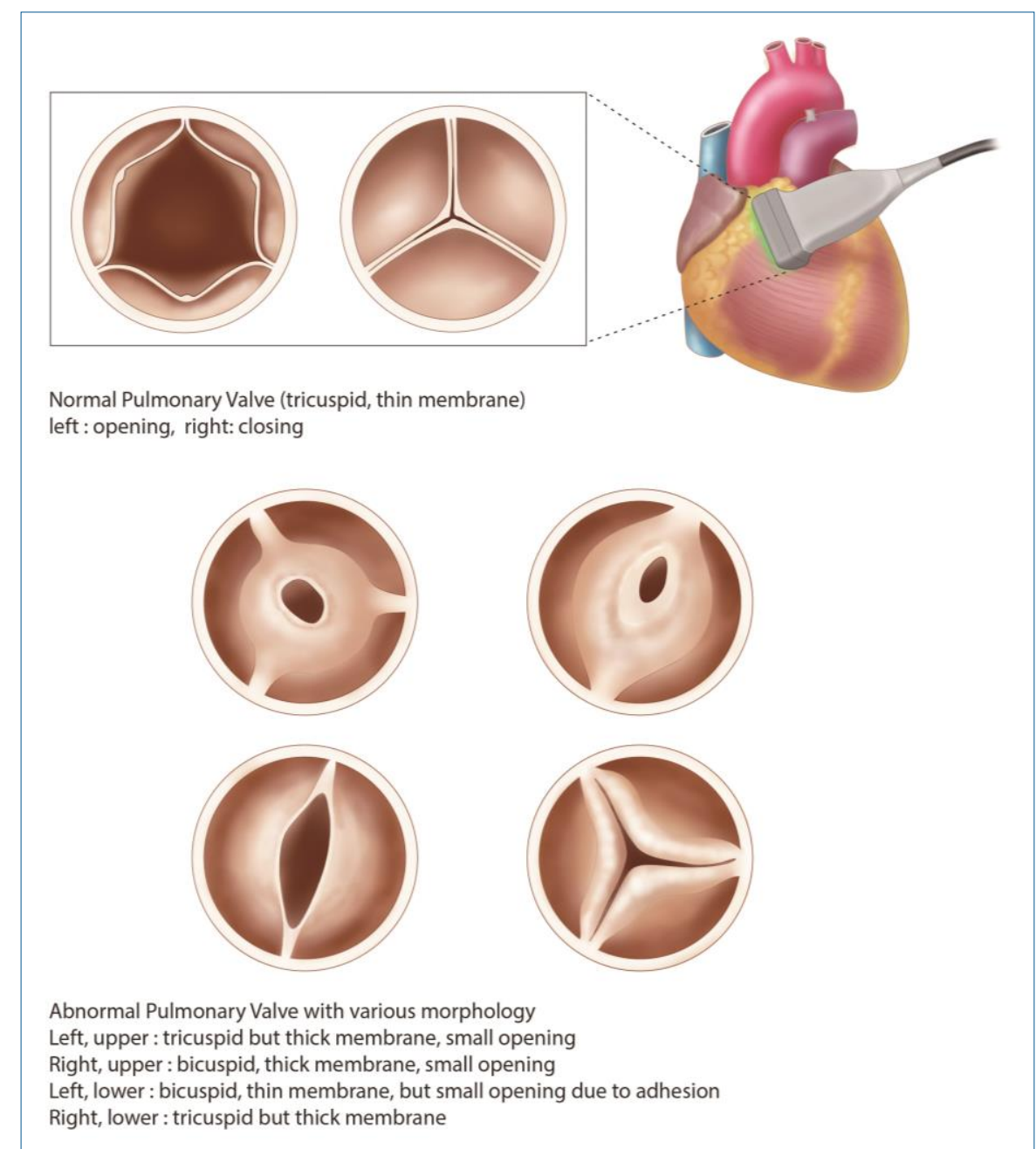

Graphical abstract. The morphology of pulmonary valve in tetralogy of Fallot (TOF). Normal pulmonary valve is tricuspid and translucent. The pulmonary valve morphology in patients with TOF is commonly abnormal: the size of the pulmonary annulus, the number of cusps, the number and location of commissures, and the number of raphe.

surface area, of which, body surface area can be computed in several ways. ${ }^{910)}$ A recent study reported the potential of the ratio of PVA to the aortic annulus and descending aorta size as a predictor of TAP repair. ${ }^{8)}$ In practice, however, there are cases where the surgical method must be changed during surgery, despite several echocardiographic findings.

We hypothesized that the morphology and tissue characteristics of PV could affect the need for TAP repair and the change of surgical method for TOF. This retrospective study aimed to determine whether echocardiographic parameters were useful for predicting the need for TAP repair during TOF surgery and identify the key factors that warranted a change in the surgical method in patients from a single hospital where pediatric patients with congenital heart disease were treated.

\section{Methods}

\section{Patients}

For the study, 62 patients who were diagnosed with TOF and underwent total correction between January 2011 and December 2018 at a single institution were enrolled. TOF was defined as: (1) pulmonary outflow tract stenosis, (2) ventricular septal defect, (3) overriding aortic root, and (4) RV hypertrophy. ${ }^{11)}$ Patients with absent PV syndrome or with an atrioventricular septal defect were excluded from the study.

Patient medical records were retrospectively reviewed to examine age, sex, body weight, echocardiographic parameters (size of PVA, number of PV cusp, aortic valve annulus, and descending aorta at the diaphragmatic level), the presence of supravalvular pulmonary stenosis (PS), history of palliation, cardiopulmonary bypass time, aortic cross clamping time, and intraoperative ratio of $\mathrm{RV}$ pressure to left ventricular pressure (PRV/LV).

\section{Echocardiographic data acquisition}

The size of the PVA, aortic valve annulus, and descending aorta at the diaphragmatic level were examined through preoperative transthoracic echocardiography (TTE) in all patients. Aortic valve and PV annular diameters were measured with magnification in parasternal long-axis views from the inner edge of the 
proximal valve insertion hinge point within the arterial root to the inner edge of the opposite hinge point. In addition, the descending aorta was measured in a subxiphoid short-axis view at the level of the diaphragm. ${ }^{12}$

\section{Total correction operation}

Total correction of TOF involved median sternotomy, followed by patch closure of the interventricular septal defect through an RV or right atrial approach, as well as enlargement of the narrow RVOT and stenotic pulmonary arteries under cardiopulmonary bypass and circulatory arrest. ${ }^{1,2)}$ If the $z$ score of the

Table 1. Patients' demographic characteristics

\begin{tabular}{lcc}
\hline Variable & $\begin{array}{c}\text { PV } \\
\text { preservation } \\
(\mathrm{n}=29)\end{array}$ & $\begin{array}{c}\text { TAP } \\
(\mathrm{n}=33)\end{array}$ \\
\hline Sex, male:female & $18: 11$ & $22: 11$ \\
Morphology of PV & $4^{*}$ & 0 \\
Tricuspid & 24 & 24 \\
Bicuspid & 1 & $9^{*}$ \\
Monocuspid & & \\
Supravalvular pulmonary stenosis & 7 & $17^{*}$ \\
Main PA & 0 & 3 \\
Peripheral PA & 6 & 9 \\
Diffuse hypoplasia of PA & & \\
Operation & & \\
Palliative operation before total correction & 0 & 4 \\
$\quad$ BT shunt & 0 & 4 \\
$\quad$ RVOT reconstruction & 2 & 1 \\
$\quad$ left PA angioplasty & 7 & 8 \\
Median age (mo) on total correction & 25 & 28 \\
Infundibullectomy & 17 & $33^{*}$ \\
RVOT incision & $19 *$ & 4 \\
Commissurotomy & & \\
Timing of reoperation & 2 & 0 \\
During total correction operation & 1 & 2 \\
During postoperative follow-up period & & \\
\hline Valesarepresed as $n$. & & \\
\hline
\end{tabular}

Values are presented as number.

$\mathrm{PV}$, pulmonary valve; TAP, transannular patch; PA, pulmonary artery; BT shunt, Blalock-Taussig shunt; RVOT, right ventricular outflow tract.

${ }^{*} P<0.05$, statistically significant difference between groups. measured PVA size was less than -2, the pulmonary arteriotomy to extend down to RVOT crossing the annulus was considered for a minimal right ventriculotomy (less than $10 \mathrm{~mm}$ ) to place the TAP.

After TOF total correction, the difference of RV pressure and pulmonary artery pressure due to residual PV stenosis was confirmed through transesophageal echocardiography (TEE), and intraoperative PRV/LV was measured through pressure monitoring. If the maximal instantaneous gradient was $>40$ $\mathrm{mmHg}$ or PRV/LV was still higher than 0.8 after the first repair with PV preservation, cardiopulmonary bypass was reinstituted for TAP repair. ${ }^{13)}$

TOF total correction was performed after a discussion between pediatric cardiologist and pediatric cardiac surgeon, and all surgeries were performed by 2 surgeons.

\section{Statistical analysis}

All statistical analyses were performed using IBM SPSS Statistics ver. 25.0 (IBM Co., Armonk, NY, USA). Data were displayed as median and range, or mean and standard deviation. Comparisons between groups were performed using the nonparametric Mann-Whitney $U$ test, 2-tailed Student $t$ test, and cross-tabulation analysis (chi-square test). $P$ value of less than 0.05 was defined as statistically significant.

\section{Ethics in research}

Patient information was retrieved and evaluated with the approval of the medical records department and Institutional Review Board at Kyungpook National University Hospital (IRB No. 2016-11-019).

\section{Results}

\section{Patient characteristics}

Patients were divided into a PV preservation group and a TAP group. The PV preservation group consisted of 29 patients (male:female $=18: 11$ ) and the median age at total correction was 7 months (range, 5-59 months). The TAP group consisted of 33 patients (male:female $=22: 11$ ) and the median age at total

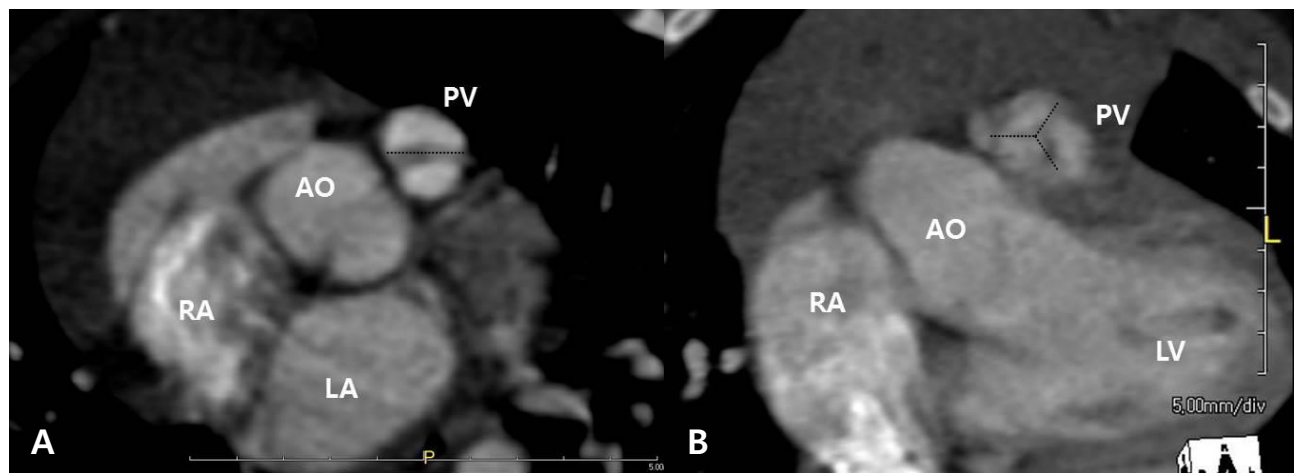

Fig. 1. Cusps of the pulmonary valve of a patient with tetralogy of Fallot on a cardiac computed tomography scan. Bicuspid pulmonary valve (A) and tricuspid pulmonary valve (B). AO, aorta; LA, left atrium; LV, left ventricle; PV, pulmonary valve; RA, right atrium. 

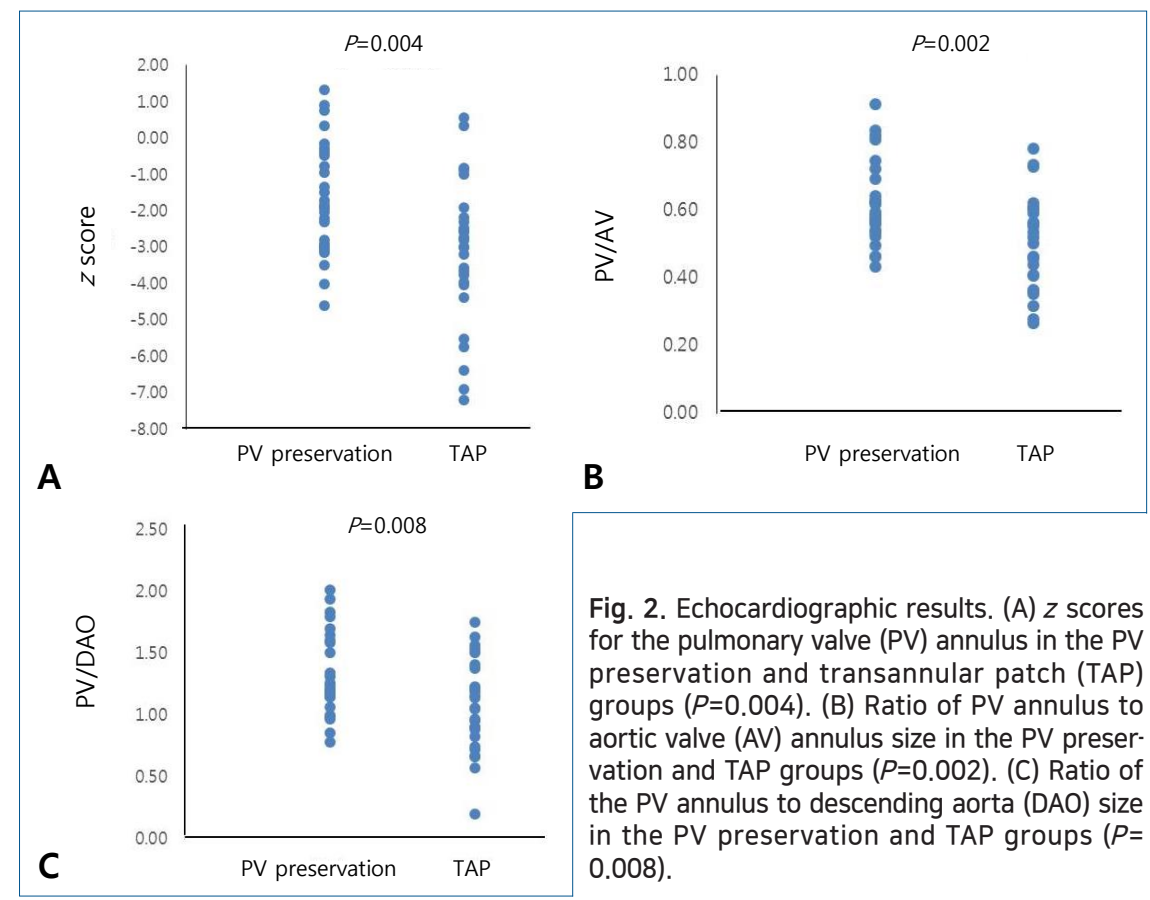

Fig. 2. Echocardiographic results. (A) $z$ scores for the pulmonary valve (PV) annulus in the PV preservation and transannular patch (TAP) groups $(P=0.004)$. (B) Ratio of PV annulus to aortic valve (AV) annulus size in the PV preservation and TAP groups $(P=0.002)$. (C) Ratio of the PV annulus to descending aorta (DAO) size in the PV preservation and TAP groups $(P=$ 0.008).

correction was 8 months (range, 1-33 months) (Table 1).

Forty-eight patients (77\%) had a bicuspid valve (Fig. 1A). One patient in the PV preservation group (1 of 29, 3\%) and 9 patients in the TAP group ( 9 of $33,27 \%$ ) had a monocuspid PV. Only 4 patients in the PV preservation group (4 of 29, 14\%) had a tricuspid PV (Fig. 1B, Table 1). The TAP group showed more patients with monocuspid PV than the PV preservation group $(P=0.011)$, and the $P V$ preservation group showed more patients with tricuspid PV than the TAP group $(P=0.027)$.

Thirteen patients in the PV preservation group (13 of $29,45 \%$ ) and 29 patients in the TAP group (29 of 33, 88\%) showed multilevel supravalvular PS (Table 1).

\section{Echocardiographic results}

The $z$ score for PVA was $-1.72 \pm 1.52$ in the PV preservation group and $-3.07 \pm 1.94$ in the TAP group $(P=0.004)$ (Fig. $2 \mathrm{~A})$. More patients in the TAP group had a $z$ score below -2, with 13 patients in the PV preservation group (13 of $29,45 \%)$ and 23 patients in the TAP group ( 23 of $33,70 \%)(P=0.048)$. The ratio of the size of PVA to aortic valve annulus was $0.62 \pm 0.12$ in the PV preservation group and $0.50 \pm 0.14$ in the TAP group $(P=0.002)$ (Fig. 2B). The ratio of the size of the PVA and descending aorta was $1.32 \pm 0.32$ in the PV preservation group and $1.07 \pm 0.36$ in the TAP group ( $P=0.008)$ (Fig. $2 \mathrm{C})$.

Receiver-operating characteristic curve analysis determined that the $z$ score cutoff value of PVA for TAP repair in our institution was -2.32 (area under the curve $[\mathrm{AUC}]=0.698 ; 95 \%$ confidence interval [CI], 0.556-0.840) with a sensitivity of $65.4 \%$ and a specificity of $73.1 \%$ (Fig. 3). If we applied a $z$ score of -2.0 , then the sensitivity was $55.0 \%$ and the specificity was $77.0 \%$. The ratio of the size of PVA to aortic valve annulus cutoff value for TAP repair was 0.56 (AUC $=0.741$; 95\% CI, 0.6070.879 ) with a sensitivity of $73.1 \%$ and a specificity of $65.4 \%$ (Fig.

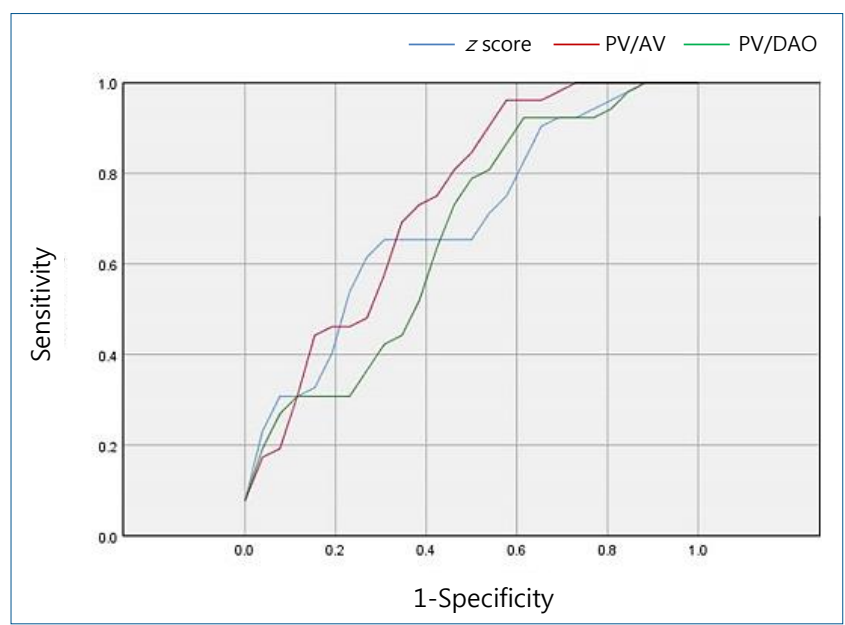

Fig. 3. Receiver operating characteristic curve analysis for the $z$ scores of the pulmonary valve (PV) annulus, ratio of the PV annulus to aortic valve (AV) annulus size, and ratio of the PV annulus to descending aorta (DAO) size cutoff value for transannular patch (TAP) repair. Blue line represents the $z$ score of the PV annulus (area under the curve $[A \cup C]=0.698 ; 95 \%$ confidence interval $[\mathrm{Cl}], 0.556-0.840)$. Red line represents the ratio of the $P V$ annulus to $A V$ annulus size (AUC $=0.741$; $95 \% \mathrm{Cl}, 0.607-0.879)$. Green line represents the ratio of the PV annulus to DAO size (AUC=0.669; $95 \% \mathrm{Cl}, 0.521-0.816)$.

3). The ratio of the size of PVA to descending aorta cutoff value for TAP repair was 1.15 (AUC $=0.669 ; 95 \%$ CI, 0.521-0.816) with a sensitivity of $69.2 \%$ and a specificity of $57.7 \%$ (Fig. 3).

\section{Surgery}

Only 2 patients in the PV preservation group (2 of 29, 7\%) underwent palliative operations, prior to total correction, but 9 patients in the TAP group ( 9 of 33, 27\%) underwent palliative operations, such as a Blalock-Taussig shunt operation, RVOT reconstruction with infundibullectomy and main pulmonary artery (MPA) angioplasty, and left pulmonary artery (LPA) angio- 
plasty prior to total correction $(P=0.110)$ (Table 1$)$.

A total of 47 patients underwent infundibulectomy during total correction, with 25 in the PV preservation group (25 of $29,86 \%)$ and 28 in the TAP group ( 28 of $33,85 \%)(P=0.880)$. All patients in the TAP group underwent RVOT incision, but only 17 patients in the PV preservation group (17 of 29,59\%) underwent RVOT incision $(P=0.001)$.

Commissurotomy was performed in 19 patients in the PV preservation group (19 of $29,66 \%)$. Of these patients, 11 had a $z$ score $<-2$, and 8 had a $z$ score $>-2$. In the TAP group, 4 patients underwent commissurotomy (4 of 33, 12\%), with 3 of them having a $z$ score $<-2$, and one having a $z$ score $>-2$. Commissurotomy was more frequently performed in the PV preservation group than the TAP group $(P=0.001)$.

$\mathrm{PRV} / \mathrm{LV}$ was measured intraoperatively in 13 patients. Of these, 7 patients were in the PV preservation group, with a median PRV/LV of 0.5 (range, 0.35-0.53). The other 6 patients were in the TAP group and the median PRV/LV was 0.59 (range, $0.37-0.79$ ). There was no significant difference between groups $(P=0.190)$.

\section{Reoperation}

Two patients for whom PV preservation was attempted underwent TAP repair due to significant PS defined by a maximal instantaneous gradient $>40 \mathrm{mmHg}$ from RVOT to main PA on the intraoperative TEE performed immediately in operating room after TOF total correction. They showed bicuspid PV, and the preoperative PVA $z$ scores were -0.83 and -1.92 , respectively. The ratios of the size of PVA to aortic valve annulus were 0.61 and 0.56 , respectively and the ratio of PVA to descending aorta was 1.53 and 1.23 , respectively. The PRV/LV was 0.57 and 0.63 , respectively.

Two patients in the PV preservation group and 2 in the TAP group had significant PS on TTE performed before discharge after TOF total correction. The preoperative PVA $z$ scores of the 2 patients in the PV preservation group were -1.85 and -0.5 , respectively. The ratios of the size of PVA to aortic valve annulus were 0.54 and 0.81 , respectively, and the ratios of the size of PVA to descending aorta were 1.26 and 1.64, respectively. They showed bicuspid PV, but PRV/LV was not measured at immediate postoperative time. One of the 2 patients underwent reoperation 10 months later and the other was lost to follow-up. The preoperative PVA $z$ scores of the 2 patients in the TAP group with significant PS were -6.39 and 0.32 . The ratios of the size of PVA to aortic valve annulus were 0.28 and 0.78 , respectively, and the ratios of the size of PVA to descending aorta were 0.57 and 1.75, respectively. They showed monocuspid and bicuspid PV, respectively, but PRV/LV was not measured at immediate postoperative time. Both patients underwent reoperation 12 months later.

One patient in the PV preservation group underwent a reoperation due to progressed PS findings (progressive infundibular stenosis, from peak flow velocity $1.5 \mathrm{~m} / \mathrm{sec}$ at the time of im. mediate postoperation to $5.5 \mathrm{~m} / \mathrm{sec}$ at the time of follow-up) on an echocardiography taken at the 17 -month postoperative follow-up.

\section{Discussion}

From the present study, we could demonstrate that PV morphology and tissue characteristics confirmed during surgery are associated with TAP repair, although the need for TAP repair during TOF total correction can be determined based on the $z$ score for PVA, and the ratio between PVA, aortic annulus and descending aorta size.

The first successful surgical correction of TOF was reported in $1954 .{ }^{14)}$ The traditional surgical method was to mitigate the obstruction of RVOT by closing the ventricular septal defect through right ventriculotomy and insertion of the TAP into the PV. ${ }^{1,2)}$ Over time, the focus of the surgical method shifted to avoiding or minimizing ventriculotomy and preserving the PV as much as possible to maintain RV function and avoid lower pulmonary regurgitation. ${ }^{3,15,16)}$

If the PV is too small to be preserved, TAP repair is unavoidable. Although TAP repair has a negative impact on long-term prognosis after TOF repair, indiscriminate preservation of the $\mathrm{PV}$ also may have a negative impact on long-term prognosis due to severe PS, RV hypertrophy, and exercise intolerance. Thus, identifying the parameters that may serve as appropriate indications for TAP repair is crucial for a successful TOF repair.

Conventionally, the $z$ score of PVA has been utilized as a predictor for the need of TAP repair. ${ }^{9,10)}$ To compute the $z$ score, the size of PVA must first be accurately measured by an experienced practitioner through echocardiography on the correct view and timing. Moreover, it should be considered that the $z$ score is influenced by the patient's height, weight, and body surface area. ${ }^{17}$

It has been reported that the ratio of the PVA to aortic annulus size can be used as a predictor of the need for TAP repair during TOF total correction in order to address the shortcomings of the $z$ score. ${ }^{8}$ The changes in the amount of blood flow from the ventricle to the great artery affect the level of growth of the artery annulus, and the ratio of the size of the PVA to the aortic annulus can be easily calculated and is not affected by other complex factors. The ratio of the size of the PVA to the aortic annulus has been reported to have significantly higher sensitivity and specificity for predicting the need for TAP repair compared to the $z$ score. ${ }^{8)}$

In the present study, the $z$ score of the PVA and the ratio between PVA, aortic annulus, and descending aorta size showed statistically significant differences between PV preservation and TAP group. However, although echocardiographic parameters were statistically effective in the prediction of the need for TAP repair, their sensitivity and specificity will not be completely satisfied.

A shortcoming of using echocardiographic parameters is that the anatomy and characteristics of the PV are not considered. 
The $z$ score is a simple index of the normalization of the PVA size and it does not indicate the degree of cusp dysplasia or suitability of the orifice. Moreover, the ratio of the PVA to the aortic annulus size and the ratio of the PVA to the descending aorta size do not reflect the characteristics of the PV, as the only size of the PVA is used in these ratios. In the present study, $58 \%$ of patients who underwent commissurotomy in the PV preservation group showed $\mathrm{PV} z$ score $<-2$. This may mean that, even though the patients had a small PV, the valve morphology and characteristics were more natural. We could find several evidences from the present study; that PV preservation group showed more tricuspid valve morphology and less supravalvar PS; that commissurotomy was higher in PV preservation group, and; that RVOT incision was higher in TAP group. Thus, solely depending on the echocardiographic parameters during TOF total correction surgery may lead to an incorrect judgment, as well as inadequate surgical results.

The postoperative RV/LV pressure is an indicator of surgical outcome, ${ }^{18,19)}$ where postoperative RV/LV pressure greater than 0.5 is associated with negative outcomes. ${ }^{16)}$ In the present study, the PVA $z$ score was less than -2 and the number of PV cusp was 2, when TAP repair was decided immediately after the PV preservation operation and the RV/LV pressure of these patients were all above 0.5 . These results suggest that preoperative $\mathrm{PV} z$ score alone is insufficient for determining surgical method and stresses the importance of RV/LV pressure measured immediately after surgery to determine remnant PS, confirmed on intraoperative TEE.

In addition, although there was no significant difference of the postoperative RV/LV pressure between groups, TAP group showed higher RV/LV pressure. We thought the reasons as 2 points: first, TAP group showed more MPA or peripheral PA hypoplasia, and we could not relieve diffuse PA hypoplasia from operation. Diffuse PA hypoplasia can make high RV pressure even though we do TAP repair: second, we permitted acceptable remnant PS during TAP repair to avoid progressive PR.

This study was subjected to the limitations inherent to a retrospective study using observational data of a single center. Another major limitation of our study was that it was not randomized, and there were significant differences between the baseline characteristics of the patients in the PV preservation group and TAP group.

Therefore, although various echocardiographic parameters may be used as predictors for determining the surgical method for TOF patients, the morphology and tissue characteristics of the PV should be also considered important.

\section{Conflicts of interest}

No potential conflict of interest relevant to this article was reported.

\section{References}

1. Apitz C, Webb GD, Redington AN. Tetralogy of Fallot. Lancet 2009;374: 1462-71.

2. Starr JP. Tetralogy of Fallot: yesterday and today. World J Surg 2010;34: 658-68.

3. Bouzas B, Kilner PJ, Gatzoulis MA. Pulmonary regurgitation: not a benign lesion. Eur Heart J 2005;26:433-9.

4. Lindberg HL, Saatvedt K, Seem E, Hoel T, Birkeland S. Single-center 50 years' experience with surgical management of tetralogy of Fallot. Eur J Cardiothorac Surg 2011;40:538-42.

5. Stewart RD, Backer CL, Young L, Mavroudis C. Tetralogy of Fallot: results of a pulmonary valve-sparing strategy. Ann Thorac Surg 2005;80:14318; discussion 1438-9.

6. Bacha E. Valve-sparing options in tetralogy of Fallot surgery. Semin Thorac Cardiovasc Surg Pediatr Card Surg Annu 2012;15:24-6.

7. Jonas RA. Early primary repair of tetralogy of Fallot. Semin Thorac Cardiovasc Surg Pediatr Card Surg Annu 2009:39-47.

8. Choi KH, Sung SC, Kim H, Lee HD, Ban GH, Kim G, et al. A novel predictive value for the transannular patch enlargement in repair of tetralogy of Fallot. Ann Thorac Surg 2016;101:703-7.

9. Awori MN, Leong W, Artrip JH, O'Donnell C. Tetralogy of Fallot repair: optimal z-score use for transannular patch insertion. Eur J Cardiothorac Surg 2013;43:483-6.

10. Awori MN, Finucane K, Gentles TL. Optimal normative pediatric cardiac structure dimensions for clinical use. World J Pediatr Congenit Heart Surg 2011;2:85-9.

11. Van Praagh R. The first Stella van Praagh memorial lecture: the history and anatomy of tetralogy of Fallot. Semin Thorac Cardiovasc Surg Pediatr Card Surg Annu 2009:19-38.

12. Lopez L, Colan SD, Frommelt PC, Ensing GJ, Kendall K, Younoszai AK, et al. Recommendations for quantification methods during the performance of a pediatric echocardiogram: a report from the Pediatric Measurements Writing Group of the American Society of Echocardiography Pediatric and Congenital Heart Disease Council. J Am Soc Echocardiogr 2010;23:465-95; quiz 576-7.

13. Rosenfeld HM, Gentles TL, Wernovsky G, Laussen PC, Jonas RA, Mayer $\mathrm{JE} \mathrm{Jr}$, et al. Utility of intraoperative transesophageal echocardiography in the assessment of residual cardiac defects. Pediatr Cardiol 1998;19:34651.

14. Lillehei CW, Cohen M, Warden HE, Read RC, Aust JB, Dewall RA, et al. Direct vision intracardiac surgical correction of the tetralogy of Fallot, pentalogy of Fallot, and pulmonary atresia defects; report of first ten cases. Ann Surg 1955;142:418-42.

15. Choi JY. Long-term problems in patients with totally repaired tetralogy of Fallot: a new paradigm for late management. J Korean Pediatr Soc 2003;46:627-34.

16. Sen DG, Najjar M, Yimaz B, Levasseur SM, Kalessan B, Quaegebeur JM, et al. Aiming to preserve pulmonary valve function in tetralogy of Fallot repair: comparing a new approach to traditional management. Pediatr Cardiol 2016;37:818-25.

17. Pettersen MD, Du W, Skeens ME, Humes RA. Regression equations for calculation of $\mathrm{z}$ scores of cardiac structures in a large cohort of healthy infants, children, and adolescents: an echocardiographic study. J Am Soc Echocardiogr 2008;21:922-34.

18. Chittithavorn V, Rergkliang C, Chetpaophan A, Vasinanukorn P, Sopontammarak S, Promphan W. Predicted outcome after repair of tetralogy of Fallot by postoperative pressure ratio between right and left ventricle. J Med Assoc Thai 2006;89:43-50.

19. Boni L, García E, Galletti L, Pérez A, Herrera D, Ramos V, et al. Current strategies in tetralogy of Fallot repair: pulmonary valve sparing and evolution of right ventricle/left ventricle pressures ratio. Eur J Cardiothorac Surg 2009;35:885-9; discussion 889-90. 